\title{
Variability of $22 \mathrm{GHz} \mathrm{H}_{2} \mathrm{O}$ masers in circumstellar shells
}

D. Engels

Hamburger Sternwarte, Gojenbergsweg 112, D-21029 Hamburg, Germany

\author{
A. Winnberg \\ Onsala Space Observatory, S-43992 Onsala, Sweden
}

J. Brand

Istituto di Radioastronomia, C.N.R., Via Gobetti 101, I-40129 Bologna, Italy

C.M. Walmsley

Osservatorio Astrofisico di Arcetri, Largo E. Fermi 5, I-50125 Firenze, Italy

\begin{abstract}
We have monitored different types of late-type stars in the $22 \mathrm{GHz}$ maser line of water vapor with single-dish telescopes and the Very Large Array over several years. We find that the emission pattern in the circumstellar shells of Semi-Regular (SR) and Mira variables mapped by the VLA remain stable on the scale of several years while the maser profiles from the single-dish telescopes vary strongly. Thus the strong variability is mainly due to incoherent intensity fluctuations of the individual maser lines, which have lifetimes less than a year.

In contrast to this, the variability in $\mathrm{OH} / \mathrm{IR}$ stars and M-Supergiants is more regular and is mainly a response to the long-period variations of the central star. $\mathrm{H}_{2} \mathrm{O}$ maser in many $\mathrm{OH} / \mathrm{IR}$ stars are "extinguished" most of the time, as the excitation temperature is only high enough during the phases close to the maximum of the variability.

The strength of the profile variability is decreasing as a function of the radial distance of the maser shell from the star. This gives a natural explanation for the increasing regularity of the maser variations along the sequence SR-, Mira variables, OH/IR stars, M-Supergiants.
\end{abstract}

\section{Introduction}

Ever since the first discovery of an $\mathrm{H}_{2} \mathrm{O}$ maser at $1.35 \mathrm{~cm}$ in the circumstellar shell of VY CMa the strong variability was mentioned, although the variations in this supergiant star are small compared to those in star forming regions (Knowles et al. 1969; Buhl et al. 1969). Monitoring programs were immediately started (Schwartz et al. 1974) and evidence was found that the masers varied in peak 
and integrated flux, in line profiles and possibly radial velocity (Dickinson et al. 1973). These variations were found to be correlated with the variations in the optical and in the infrared (Schwartz et al. 1974). Later observations showed the variations to be less regular (Engels et al. 1988; Cohen 1989). The first interferometric maps of the Miras R Aql and RR Aql taken at two epochs separated by $\approx 1$ year also showed dramatic changes (Johnston et al. 1985). Monitoring of $\mathrm{OH} / \mathrm{IR}$ stars had not been attempted, but the available observations indicated more regular variations than in SR and Mira variables (Engels et al. 1986). To improve the understanding of the properties of maser variability for the different types of late-type stars, we have monitored since 1990 several of them with single-dish telescopes and the VLA.

\section{Observations}

Single dish observations have been made with the Effelsberg $100 \mathrm{~m}$ and Medicina $32 \mathrm{~m}$ telescopes at typical intervals of a few months for the years 1990-1997. The spectra have a velocity resolution of $0.1-0.3 \mathrm{~km} \mathrm{~s}^{-1}$ with the noise varying between 0.1 and $5 \mathrm{Jy}$. Interferometric maps were obtained with the VLA in its A-configuration for a few representative stars at several epochs. These stars were the SR variable RX Boo, the Mira U Her, the OH/IR stars $\mathrm{OH} 39.7+1.5$ and $\mathrm{OH}$ 83.4-0.9, and the M-Supergiant VX Sgr. The smoothed spectral resolution of the VLA data is $1.32 \mathrm{~km} \mathrm{~s}^{-1}$, the angular resolution $\approx 80 \mathrm{mas}$, and the rms sensitivity $\leq 30 \mathrm{mJy}$.

In the following we discuss the results for $\mathrm{U}$ Her and $\mathrm{OH} 83.4-0.9$ as representative cases. First results on RX Boo were given by Engels et al. (1992), and on $\mathrm{OH} 39.7+1.5$ by Engels et al. (1997).

\section{U Her}

$\mathrm{U}$ Her is a well-observed Mira variable with a period of 406 days. It exhibits strong maser emission not only in $\mathrm{H}_{2} \mathrm{O}$, but also in $\mathrm{OH}$ and $\mathrm{SiO}$. Maps in the $1.35 \mathrm{~cm}$ line were obtained in 1990 February and June, in 1991 October and 1992 December. In 1991 the VLA was in BnA-configuration (intermediate between $\mathrm{A}$ and $\mathrm{B}$ ) which degraded the east-west resolution to $\approx 300$ mas. Generally the maser spots were not resolved and individual spots were identified by decomposition of the channel maps with multiple gauss fits. The maps, containing all maser spots for a given epoch, and two typical single-dish spectra are shown in Fig. 1, and the light curve for the integrated flux in Fig. 2. The maps were shifted tentatively to a common origin, assuming that the masers lie in a ring indicated by the circle drawn in each map. We also tentatively assume that the star is located at the center of the ring. The size of the symbols is scaled with intensity and a few components are labeled by their velocities.

Maser spots were detected between -22 and $-8 \mathrm{~km} \mathrm{~s}^{-1}$, with two dominating components at $\approx-18$ and $\approx-15 \mathrm{~km} \mathrm{~s}^{-1}$. To distinguish between them in the maps all components with $\mathrm{v} \leq-16 \mathrm{~km} \mathrm{~s}^{-1}$ were drawn as open symbols. The stellar radial velocity is at $\approx-14 \mathrm{~km} \mathrm{~s}^{-1}$, so that almost all of the emission is blue shifted. In the period 1990-1997, except for about a year in 1991/92, the $-15 \mathrm{~km} \mathrm{~s}^{-1}$ component was always the strongest. The maps show most of 

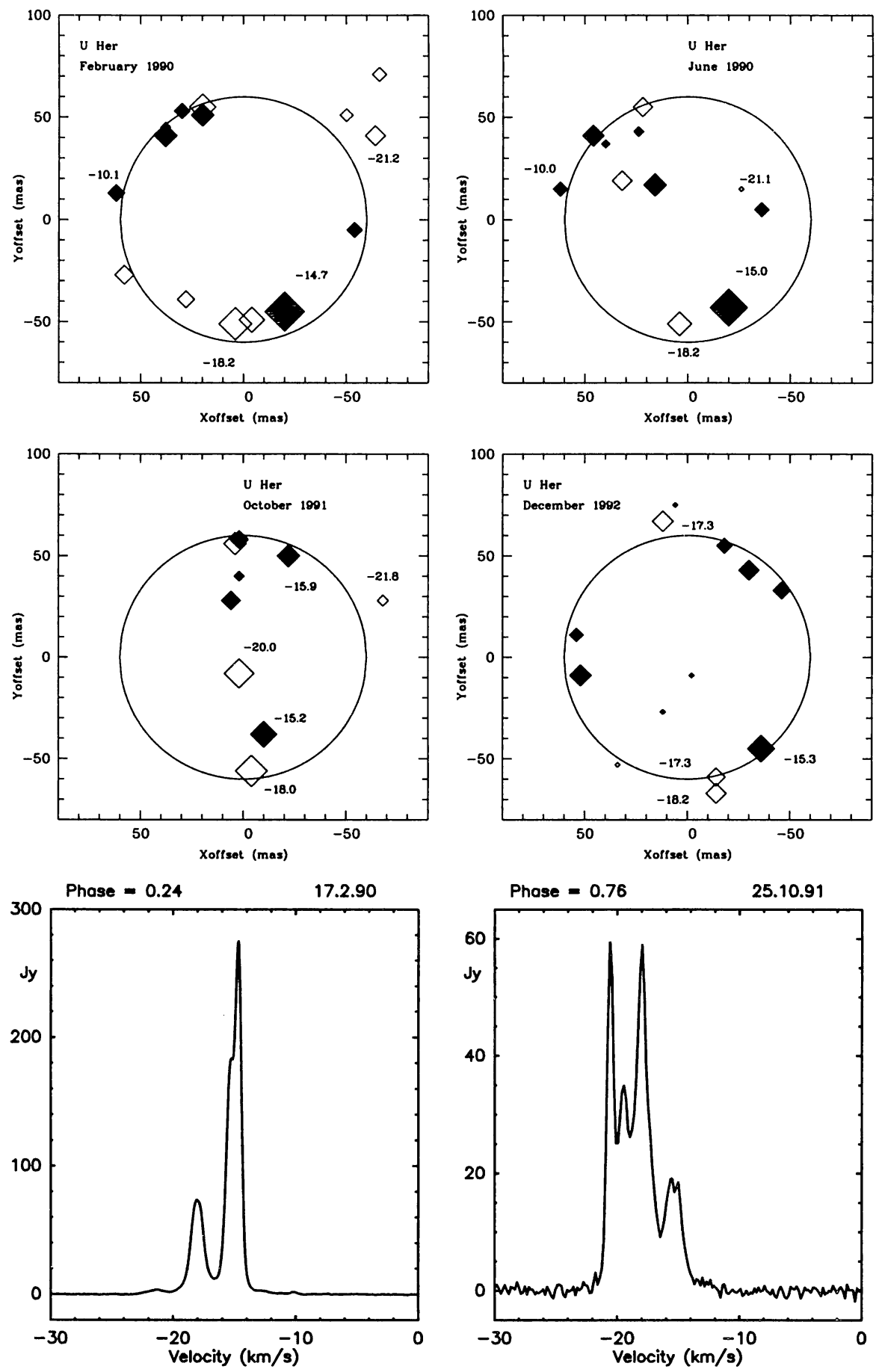

Figure 1. U Her. Maps and representative single-dish spectra. See text for the meaning of the symbols. 

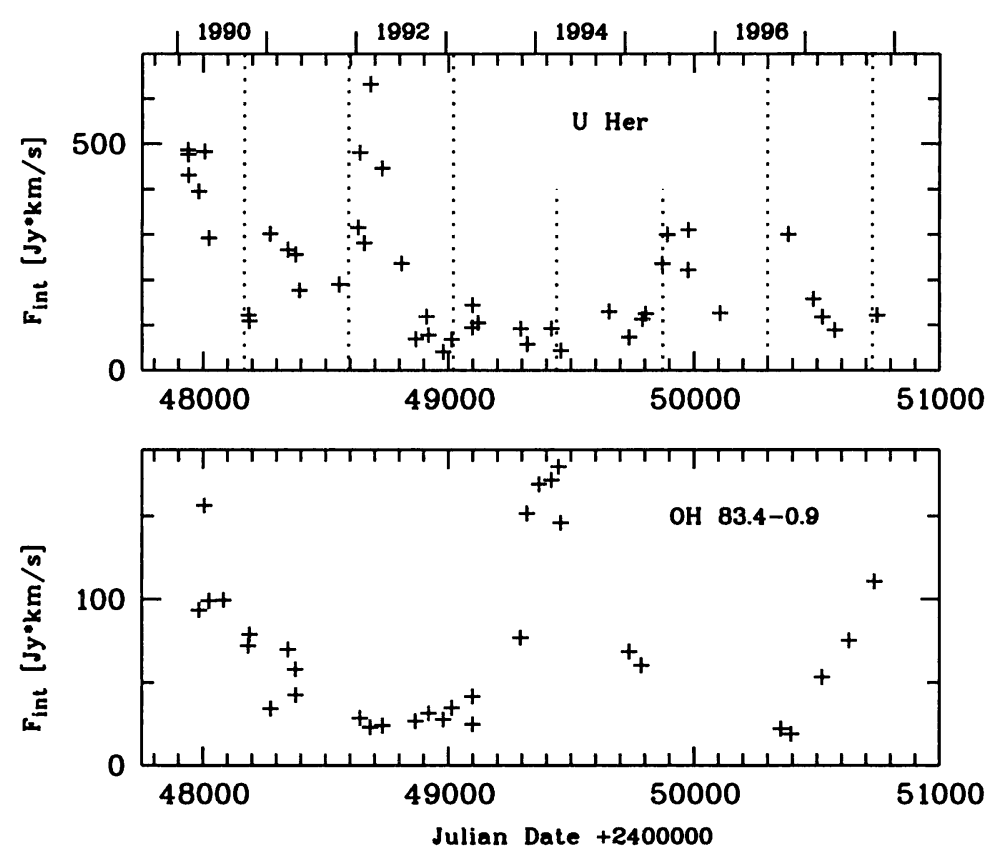

Figure 2. Light curves of the integrated maser flux. The vertical lines mark the maxima of the optical U Her lightcurve.

the individual maser spots to be located in a ring of $\approx 60$ mas radius, which corresponds to $21 \mathrm{AU}$ at the star distance of $350 \mathrm{pc}$. All four maps show the two dominating components to be located in the southern part of the ring. In the two maps obtained in 1990 within four months, the maser components detected are almost identical. The differences between the maps in Fig. 1 are due to blending of components and intensity variations. This leads to apparent positional changes (cf. the $-21 \mathrm{~km} \mathrm{~s}^{-1}$ component) and to the presence of several spots in only one epoch. The 1991 map was taken while the emission was dominated by features with $v \leq-18 \mathrm{~km} \mathrm{~s}^{-1}$. Because of the degraded resolution we have no information of the east-west extent of the shell. The very blue maser line came from about the center of the map and could be amplified light of the central star. The 1992 map shows again the ring-like structure as in 1990, but it is not possible to identify individual spots common to both epochs. Interestingly the strongest components still come from the southern part of the ring but shifts in the velocity indicate that these are also different masers from those in 1990.

This star was also observed with MERLIN in 1985 (Yates \& Cohen 1994), with the VLA in 1983 and 1988 (Lane et al. 1987; Bowers \& Johnston 1994), and with the VLBA in 1995 by Marvel (1997). Comparing our maps with these ones shows that at least between 1988 and 1995 the main feature, the location of the strongest maser in the south of the spatial distribution, was maintained. During the early VLA and MERLIN observations only emission over a rather small velocity range was detected, precluding conclusions on the shell structure. The U Her observations show that the individual maser spots have a lifetime of 

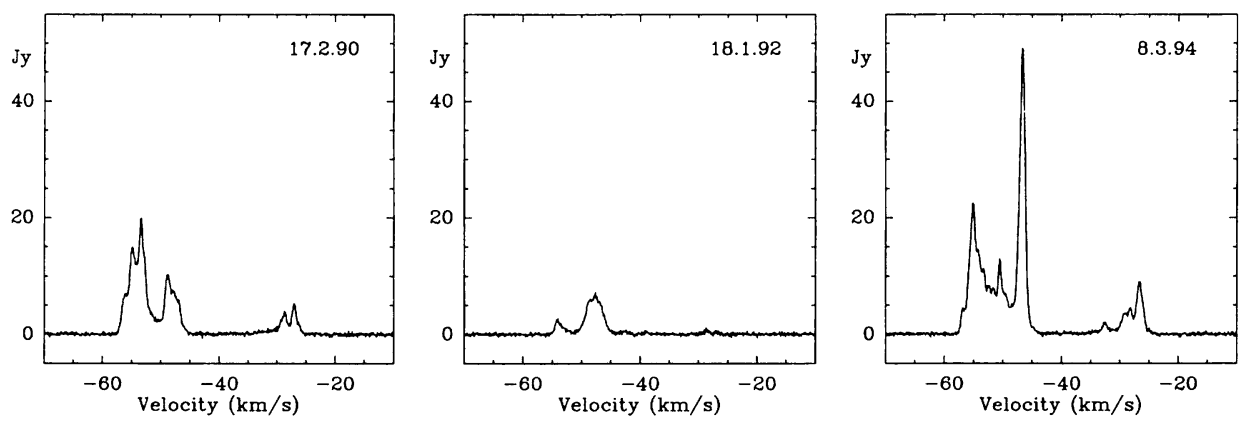

Figure 3. Spectra of $\mathrm{OH} 83.4-0.9$ during the maxima in 1990 and 1994 and during the minimum in 1992.

at least several months, while the general structure of the maser shell remains stable over $\geq 7$ years. The crossing time through the maser shell is of the order of 10 years and more. Thus we may expect to observe changes in the map structure in the future.

\section{OH 83.4-0.9}

This is an $\mathrm{OH} / \mathrm{IR}$ star with a period of $\approx 1400$ days. Examples of the doublepeaked $\mathrm{H}_{2} \mathrm{O}$ maser spectra, which are typical for $\mathrm{OH} / \mathrm{IR}$ stars, are shown in Fig. 3. The light curve (Fig. 2) shows a regular variation with the period of the star. Intensity fluctuations of individual maser lines are less pronounced than in UHer. Two VLA maps taken in 1990 did not resolve the maser shell but a size of $\approx 30$ mas can be inferred from the positional shifts with velocity. A new VLBA map that we made in 1998 confirms this estimate. The distance of $\mathrm{OH} 83.4-0.9$ is $2-4 \mathrm{kpc}$ yielding a shell radius of $60-120 \mathrm{AU}$. This compares well with the maser shell radius obtained by Engels et al (1997) for $\mathrm{OH} 39.7+1.5$ and shows that the maser shells of $\mathrm{OH} / \mathrm{IR}$ stars are $\approx 5$ times larger than in $\mathrm{SR}$ and Mira variables.

\section{Discussion}

So far, the discussion of $\mathrm{H}_{2} \mathrm{O}$ maser variability was dominated by early monitoring programs including mostly Miras and SR variables. In these stars with low mass loss rates $\left(<10^{-6} \mathrm{M}_{\odot} / \mathrm{yr}\right)$ the maser shell is located at $\leq 50 \mathrm{AU}$ (see also Bowers \& Johnston 1994), within the zone of radial acceleration of the shell. There masers are beamed preferentially in a tangential direction (Chapman \& Cohen 1985), leading to profiles peaked close to the stellar radial velocity. Irregular intensity fluctuations prevail, leading to a rather weak correlation with the optical lightcurve. The lifetime of individual maser spots appears to be $\leq 1$ year, while the distribution of maser sites in the shell survives several years.

Bowers \& Johnston (1994) found variations of the maser shell sizes comparing their maps to earlier interferometric data. We did not find such variations for RX Boo and U Her. They can be mimicked by varying sensitivities of the maps 
as the weaker emission spots tend to have larger radial distances and might not be detected close to minimum phase.

In $\mathrm{OH} / \mathrm{IR}$ stars, which have higher mass loss rates $\left(>10^{-5} \mathrm{M}_{\odot} / \mathrm{yr}\right)$, the maser shell is shifted to larger distances, at which the final expansion velocity is (almost) reached. Radial beaming leads to double-peaked profiles. In one case, $\mathrm{OH} 39.7+1.5$, we discovered a "mode switch" from radial to tangential beaming during minimum phase (Engels et al. 1997). Regular intensity variations following the bolometric variations of the star lead to rather regular maser lightcurves. Often the masers are "extinguished" over a considerable fraction of the variation cycle, as the excitation temperatures seem to be high enough only during the phases close to maximum. The stability of the maser profiles over several variability cycles suggests, that the shell structure will be maintained over longer timescales than in Miras and SR variables.

M-Supergiants have even higher mass loss rates and luminosities, and larger $\mathrm{H}_{2} \mathrm{O}$ maser shells than $\mathrm{OH} / \mathrm{IR}$ stars (Richards \& Yates 1998). Their variability properties are similar to those of $\mathrm{OH} / \mathrm{IR}$ stars. SR variables, Miras, OH/IR stars, and M-Supergiants form a sequence of increasing maser shell sizes and decreasing strength of irregular variability.

Acknowledgments. We acknowledge the support by the Deutsche Forschungsgemeinschaft (Re $353 / 25$, En 176/2) and by the Swedish Natural Science Research Council. The observations were partially obtained at the VLA, which is part of the NRAO operated by Associated Universities Inc., under cooperative agreement with the National Science Foundation of the USA.

\section{References}

Bowers P.F., Johnston K.J., 1994, ApJS 92, 189

Buhl D., Snyder L.E., Schwartz P.R., Barrett A.H., 1969, ApJ 158, L97

Chapman J.M., Cohen R.J., 1985, MNRAS 230, 415

Cohen R.J., 1989, Rep. Prog. Phys. 52, 881

Dickinson D.F., Bechis K.P., Barrett A.H., 1973, ApJ 180, 831

Engels D., Schmid-Burgk J., Walmsley C.M., 1986, A\&A 167, 129

Engels D., Schmid-Burgk J., Walmsley C.M., 1988, A\&A 191, 283

Engels D., Winnberg A., Brand J., Walmsley C.M., 1992, in Astrophysical Masers, eds. A. Clegg and G. Nedoluha, p. 403

Engels D., Winnberg A., Walmsley C.M., Brand J., 1997, A\&A 322, 291

Johnston K.J., Spencer J.H., Bowers P.F., 1985, ApJ 290, 660

Knowles S.H., Mayer C.H., Cheung A.C., Rank D.M., Townes C.A., 1969, Science 163,1055

Lane A.P., Johnston K.J., Bowers P.F., Spencer J.H., Diamond P.J., 1987, ApJ 323, 756

Marvel K., 1997, PASP 109, 1286

Richards A.M.S., Yates J.A., 1998, Irish Astr. J. 25, 7

Schwartz P.R., Harvey P.M., Barrett A.H., 1974, ApJ 187, 491

Yates J.A., Cohen R.J., 1994, MNRAS 270, 958 